\title{
Review
}

\section{Guardian ancestry: fly p53 and damage-inducible apoptosis}

\author{
W Nordstrom ${ }^{1}$ and JM Abrams ${ }^{\star, 1}$ \\ 1 Department of Cell Biology, University of Texas, Southwestern Medical Center, \\ 5323 Harry Hines Boulevard, Dallas, Texas 75390-9039, USA \\ * Corresponding author: JM Abrams, University of Texas, Southwestern \\ Medical Center, 5323 Harry Hines Boulevard, Dallas, Texas 75390-9039, USA. \\ E-mail John.Abrams@utsouthwestern.edu
}

Received 18.7.00; revised 20.8.00; accepted 23.8.00

Edited by S Kumar

\begin{abstract}
The tumor suppressor, p53, is among the most commonly mutated genes in human cancers. Recent reports describe shared and divergent properties of a Drosophila p53 homolog Dmp53. Like its mammalian counterpart, Dmp53 also functions in damage-induced cell death. In this model system, the apoptosis activator reaper has emerged as an important target gene. Together with the wealth of genomic data available in Drosophila, continued studies on Dmp53 promise new insights into the regulation and function of this important gene family. Cell Death and Differentiation (2000) 7, 1035-1038.
\end{abstract}

Keywords: Drosophila; apoptosis; p53

Abbreviations: CDKs, cyclin-dependent kinases; IAP, inhibitor of apoptosis protein; $\mathrm{Rb}$, retinoblastoma; $\mathrm{RE}$, responsive element; SAM, sterile alpha motif

\section{Introduction}

The p53 tumor suppressor acts as a cellular guardian, responding to genotoxic stress by either arresting the cell cycle and promoting DNA repair or eliminating potentially damaged cells via apoptosis. Not surprisingly, disabling such a safeguard appears to be a common route toward oncogenic transformation as a predominance of human cancers are associated with mutations at the p53 locus. p53 functions as a transcription factor and several targets connected to cell cycle regulation, DNA repair and apoptosis have been characterized. The precise mechanisms that specify these distinct responses, however, remain largely ill defined.

The recent identification by Brodsky et al., Ollman et al., and Jin et al. of a Drosophila p53 homolog, Dmp53, provides a new and valuable avenue for dissecting p53's function in development and damage responses. ${ }^{1-3}$ The 385 amino acid Dmp53 protein shares properties in four regions previously characterized for their discrete functions in mammalian p53. These domains divide p53 into a transcriptional activation domain at the N-terminus, a DNA binding domain, an oligomerization domain and basic domain at the C-terminus. Such domains were identified in Dmp53 with the DNA binding domain representing the strongest area of homology $(43 \%$ similarity). More significantly, of the six sites most frequently found mutated in mammalian tumors, Dmp53 is identical in four and similar in the remaining two. Of these four identical residues, two have been shown to directly contact DNA from mammalian studies. Among six other residues involved in DNA binding, four are identical and one is similar in Dmp53. In addition, the four residues that contact zinc are also conserved.

\section{Phylogeny}

As the most evolutionarily remote representative of the p53 family, Drosophila p53 can provide insights into the gene family's ancestry. Prior to Dmp53's identification, squid was the most distantly related species for which a p53 homolog was isolated. However, squid p53 is more similar to the human p63/p73 subfamily of p53 because all contain a Sterile Alpha Motif (SAM) domain. This led to the proposal that extant p53 family members arose from a p73-like ancestor. ${ }^{4}$ Dmp53 is equally distant from human $p 53$, human $p 73$, and squid p53, but lacks a SAM domain. With no p63/p73 homologs identified in the near complete Drosophila genome, Ollman et al. suggested that the p53 gene family originated from a p53-like ancestor and that the p63/p73 subfamily evolved after the divergence between vertebrate and invertebrate lineages. Current phylogenic relationships are at odds with this proposal, however, since the common ancestor of insects and molluscs is certainly more recent than the divergence between protostomes and deuterostomes. A more likely alternative is that the common ancestor of invertebrates and vertebrates contained both $p 53$ and p73-like genes and that p73-like genes may have been selectively lost in the arthropod lineage. Moderate support for this scenario is suggested from lineage algorithms that use only the DNA binding domain of $\mathrm{p} 53$ family members. If only this domain is considered to be the evolutionary unit, Dmp53 shows a slightly closer resemblance to the members p63/p73 subfamily (see Table 1). This result might support the possibility of a p73-like ancestor and also implicates the existence of variants with and without the SAM domain in the common ancestor. An important missing piece of the puzzle, of course, is whether or not other invertebrate genomes contain both p73 and p53-like genes. In light of this, recent reports of p53 family members from the clam suggest that molluscs do indeed have p53 like genes with and without SAM domains. ${ }^{5}$ Clearly, any definitive statements about p53's evolutionary origin requires more complete genomic information from a variety of species representing several metazoan radiations.

Although Drosophila p53 ranks as the most divergent member of the family yet described, numerous studies established that Dmp53 shares properties with its 
Table 1 Sequence relationships between the DNA binding domains of $p 53$ family genes

\begin{tabular}{cccccc}
\hline & $\begin{array}{c}\text { Human } \\
\text { p53 }\end{array}$ & $\begin{array}{c}\text { Human } \\
\text { p73 }\end{array}$ & $\begin{array}{c}\text { Squid } \\
\text { p53 }\end{array}$ & $\begin{array}{c}\text { Clam } \\
\text { p53 }\end{array}$ & $\begin{array}{c}\text { Clam } \\
\text { p73 }\end{array}$ \\
\hline Dmp53 & $25 \%$ & $29 \%$ & $29 \%$ & $32 \%$ & $32 \%$ \\
\hline
\end{tabular}

THe DNA binding domain of Drosophila p53 (Genbank Accession: AF224713) was compared against human p53 (NP 000537), human p63 (AF075433), human p73 (NM 005427), squid (U43595), Xenopus (X77546), zebrafish (U60804), clam p53 (AF253323), and clam p73 (AF253324) using Blast 2 (Basic Local Alignment Search Tool). ${ }^{36}$ Identity scores are shown

mammalian counterparts. Both the full-length protein, as well as isolated domains predicted from protein alignment algorithms, were tested in numerous contexts. In two-hybrid experiments, the $\mathrm{N}$-terminal region conferred transcriptional activation while the $\mathrm{C}$-terminal region promoted oligomerization. Site specific DNA binding was demonstrated using mobility shift assays, ${ }^{2,3}$ one-hybrid tests and cell culture transfections. ${ }^{1}$ Together, these analyses established that Dmp53 can bind to human p53 binding sites.

Functional studies also validated an orthologous role in damage inducible apoptosis. Like its mammalian counterpart, overexpression of Dmp53 triggers ectopic apoptosis and variants that are disrupted for DNA binding activity exert dominant negative effects. In the developing wing disc, dominant-negative Dmp53 expression blocked radiation-induced apoptosis but had no apparent affect on normal development. Similar observations were documented in transfection experiments where mutant Dmp53 poisoned transactivation by the wild-type protein. A likely explanation for these observations is that the mutant Dmp53, like other members of this family, forms inactive tetrameric complexes with the wild-type protein.

\section{New insights}

The availability of a p53 homolog in the Drosophila model system holds the potential for rapid elucidation of novel pathways controlled by p53. Such promise has already been partially realized with the identification of reaper as a likely p53 transcriptional target by Brodsky and colleagues. reaper is a pro-apoptotic gene whose expression is governed by developmental signals and damaging stimuli. ${ }^{6}$ Later studies showed that induction of reaper by ionizing radiation is under transcriptional control, implicating an upstream factor satisfying a role orthologous to p53 in mammals. ${ }^{7}$ Further delineation of reaper's regulatory region identified a minimal radiation responsive element that matched the consensus p53 responsive element. The consensus p53 binding element (p53 RE) consists of two repetitions of a 10 mer. While in vitro characterizations of the p53 RE suggest a tolerance for up to a $13 \mathrm{bp}$ separation between the 10 mers, native in vivo targets such as those found upstream of the human target genes $m d m-2^{8}$ and $p 21 / W A F 1^{9}$ typically contain adjacent sites. Like these mammalian targets, the radiation responsive element upstream of reaper also contains adjacent sites. In this case, two mismatches exist at the outermost nucleotides of the p53RE, thus preserving an invariant 18 base pair core.
The identification of Dmp53 as a potential regulator of reaper expression provides a satisfying link between genotoxic stress and apoptosis in Drosophila. In this model system, reaper, hid, and grim respond differentially to various apoptotic stimuli including ionizing radiation. ${ }^{10}$ These promote caspase activation by acting positively through Dark, the Apaf-1/Ced-4 homolog, ${ }^{11-13}$ and inhibiting anti-apoptotic functions such as the inhibitor-ofapoptosis proteins (IAPs). ${ }^{14,15}$ Released from the inhibiting grip of IAPs, caspases are then presumably free to activate downstream caspases, committing the cell to an apoptotic fate (Figure 1).

The reaper protein, at only 65 amino-acids in length, shares no obvious sequence similarity with genes outside of other insects. The possible existence of a mammalian reaper homolog, however, is supported by the observations that Reaper can induce apoptosis in mammalian cell culture systems as well as bind mammalian IAPs. ${ }^{16-19}$ Reaper's small size has strained the limits of algorithms used to identify prospective homologs in genomic databases. The connection between p53 and reaper in flies may assist computational searches for mammalian reaper homologs. Likewise, it will be interesting to see whether Smac/Diablo, a recently identified inhibitor of IAPS (with no obvious homology to reaper $)^{20,21}$ might be responsive to p53.

\section{Missing players}

As mentioned above, functional studies on DmP53 in the eye and wing discs established profound effects upon apoptosis but uncovered no apparent affect upon cell-cycle progression. While it is possible that both primordial wing and retinal tissues are atypically insensitive to p53 induced arrest, it is more likely that Dmp53 simply does not engage the cell cycle machinery in the same way that mammalian members of this family do. This is a surprising result given the extensive cell cycle arrest properties documented for mammalian p53 proteins (reviewed $\mathrm{in}^{22}$ ) and the conservation of orthologous cell cycle regulators in the fly. In mammals, transactivation of p21 by p53 defines a major pathway that inhibits the activity of cyclin-dependent kinases (CDKs) and prevents phosphorylation of the retinoblastoma $(\mathrm{pRb})$ protein. ${ }^{23} \mathrm{p} 21$ can also trigger G1 arrest in Drosophila ${ }^{24}$ but despite the preservation of this checkpoint pathway, existing evidence suggests that Dmp53 does not appear to use it (although transactivation of fly p21 could possibly occur without triggering a checkpoint arrest).

Similarly, the ATM/Chk1 pathway is conserved in Drosophila $^{25}$ and is required for G2/M arrest in irradiated wing discs. ${ }^{26}$ In mammals, ATM phosphorylates p53 on serines located in serine-glutamate pairs at positions 15 and $37 .{ }^{27-29}$ Dmp53 contains a serine 4-glutamate 5 site which could serve as a phosphorylation target for Mei-41, the Drosophila ATM homolog. One model for ATM function suggests that phosphorylation of $\mathrm{p} 53$ by this kinase impairs binding and disposal by Mdm2. ${ }^{30}$ Relating this scenario to Dmp53 is problematic, however, considering that orthologs of Mdm2 and its upstream regulator p19ARF are absent from the fly genome. ${ }^{26}$ Perhaps equally important is the fact that key residues essential for binding to Mdm2 by p53 are absent in Dmp53. Thus, it is likely that alternate 


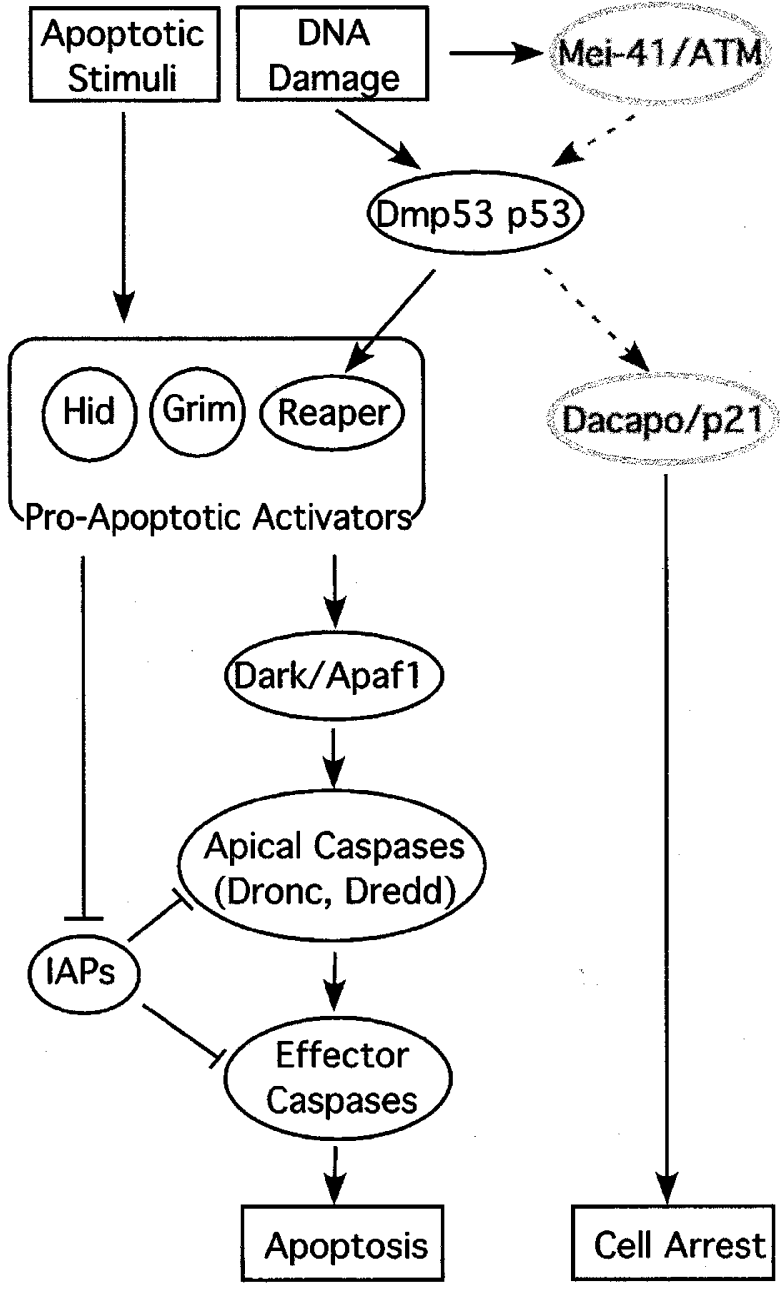

Figure 1 Regulation of apoptosis by Dmp53. In response to DNA damage, both mammalian p53 and its Drosophila ortholog (Dmp53) can respond by initiating apoptosis. In Drosophila, Dmp53 transactivates expression of reaper, one of three closely linked pro-apoptotic genes that respond to various apoptotic stimuli. Reaper leads to the activation of apical caspases possibly through the inhibition of IAPs (inhibitor of apoptosis proteins) and/or activation of the Apaf-1 homolog, Dark. ${ }^{11}$ The apoptotic program continues with the subsequent activation of downstream effector caspases. As indicated in gray, Mei-41 and Dacapo, the fly homologs of ATM and p21, respectively, have demonstrated involvement in cell cycle arrest. Unlike mammalian p53, however, no role for Dmp53 in cell cycle arrest has been detected by either Brodsky et al., Ollman et al., or Jin et al.

mechanisms connecting genotoxic stress signals to Dmp53 action probably exist.

Future studies will continue to elucidate the extent to which the insect p53 pathways have diverged from vertebrates. It remains to be seen, for instance, whether Dmp53 has been completely divested of a role in cell cycle arrest. The limited results available thus far do not rule out the possible necessity of cooperative factors or 'co-signals' in promoting cell cycle arrest. Likewise, these interpretations rely upon dominant negative proteins and a tenuous presumption that Dmp53's homotetramerization is a conserved requirement for its cell cycle arrest function. The production of single gene mutations in Dmp53 should help address these and other important issues. Given $\sim 550$ million years of evolutionary distance separating the two lineages, it is quite likely that significant upstream and downstream differences will emerge. In this regard it will be interesting to see if homologs for mammalian p53 targets such as $p 21^{31}$ and $b a x^{32}$ are targets for Dmp53 as well.

\section{Future directions}

Studies of fly counterparts to known participants in the mammalian p53 response pathways promise to reveal core elements in relevant damage responses. At the same time, points of divergence, such as the absence of Mdm-2, should highlight which features of p53 regulation are distinct in mammals. Beyond investigating known Drosophila homologs, the nearly complete genome sequence for both organisms provides exciting new possibilities for elaborating as yet undiscovered pathways. Already, high throughput analysis concentrating on mammalian genes regulated by $\mathrm{p} 53$ has produced a wealth of data by which to compare and contrast such efforts in Drosophila. ${ }^{33-35}$ Toward this end the p53 responsive element identified in the reapergene may serve as a valuable tool for identifying genes involved in the damage response.

\section{References}

1. Brodsky MH, Nordstrom W, Tsang G, Kwan E, Rubin GM and Abrams JM (2000) Drosophila p53 binds a damage response element at the reaper locus. Cell 101: $103-113$

2. Ollmann M, Young LM, Di Como CJ, Karim F, Belvin M, Robertson S, WhittakerK, Demsky M, Fisher WW, Buchman A, Duyk G, Friedman L, Prives C and KopczynskiC (2000) Drosophila p53 is a structural and functional homolog of the tumor suppressor p53. Cell 101: $91-101$

3. Jin S, MartinekS, JooWS, Wortman JR, Mirkovic N, Sali A, Yandell MD, Pavletich NP, Young MW and Levine AJ (2000) Identification and characterization of a p53 homologue in Drosophila melanogaster. Proc. Natl. Acad. Sci. USA 97: 7301 7306

4. Kaghad M, Bonnet H, Yang A, Creancier L, Biscan JC, Valent A, Minty A, Chalon P, Lelias JM, Dumont X, Ferrara P, McKeon F and Caput D (1997) Monoallelically expressed gene related to P53 at $1 \mathrm{p} 36$, a region frequently deleted in neuroblastoma and other human cancers. Cell 90: 809-819

5. Van Beneden RJ, Walker CW and Laughner ES (1997) Characterization of gene expression of a 53 homologue in the soft-shell clam (Mya arenaria). Mol. Marine Biol. Biotechnol. 6: 116-122

6. White K, Grether M, Abrams JM, Young L, Farrell K and Steller H (1994) Genetic control of programmed cell death in Drosophila. Science 264: 677-683

7. Nordstrom W, Chen P, Steller H and Abrams JM (1996) Activation of the reaper gene during ectopic cell killing in drosophila. Dev. Biol. 180: 213-226

8. Wu X, Bayle JH, Olson D and Levine AJ (1993) The p53-mdm-2 autoregulatory feedback loop. Genes Dev. 7: 1126-1132

9. El-Deiry WF, Tokino T, Velculescu VE, Levy DB, Parsons R, Trent JM, Lin D, Mercer WE, Kinzler KW and Vogelstein B (1993) WAF1, a potential mediator of p53 tumor suppression. Cell 75: 817-825

10. Abrams JM (1999) An emerging blueprint for apoptosis in Drosophila. Trends Cell Biol. 9: 435-440

11. Rodriguez A, Oliver H, Zou H, Chen P, Wang XD and Abrams JM (1999) Dark is a Drosophila homologue of Apaf-1/CED-4 and functions in an evolutionarily conserved death pathway. Nature Cell Biol. 1: 272-279

12. Zhou L, Song ZW, Tittel J and Steller H (1999) HAC-1, a Drosophila homolog of APAF-1 and CED-4 functions in developmental and radiation-induced apoptosis. Mol. Cell 4: 745-755 
13. Kanuka H, Sawamoto K, Inohara N, Matsuno K, Okano H and Miura M (1999) Control of the cell death pathway by Dapaf-1, a Drosophila Apaf-1/CED-4-related caspase activator. Mol. Cell 4: 757-769

14. Wang SL, Hawkins CJ, Yoo SJ, Muller HAJ and Hay BA (1999) The Drosophila caspase inhibitor DIAP1 is essential for cell survival and is negatively regulated by HID. Cell 98: 453-463

15. Goyal L, McCall K, Agapite J, Hartwieg E and Steller H (2000) Induction of apoptosis by Drosophila reaper, hid and grim through inhibition of IAP function. EMBO J. 19: 589-597

16. Evans EK, Kuwana T, Strum SL, Smith JJ, Newmeyer DD and Kornbluth S (1997) Reaper-induced apoptosis in a vertebrate system. EMBO J. 16: 7372-7381

17. McCarthy JV and Dixit VM (1998) Apoptosis induced by Drosophila Reaper and Grim in a human system attenuation by inhibitor of apoptosis proteins (Ciaps). J. Biol. Chem. 273: 24009-24015

18. Thress K, Evans EK and Kornbluth S (1999) Reaper-induced dissociation of a Scythe-sequestered cytochrome c-releasing activity. EMBO J. 18: 5486-5493

19. Thress K, Henzel W, Shillinglaw W and Kornbluth S (1998) Scythe a nove reaper-binding apoptotic regulator. EMBO J. 17: 6135-6143

20. Verhagen AM, Ekert PG, Pakusch M, Silke J, Connolly LM, Reid GE, Moritz RL, Simpson RJ and Vaux DL (2000) Identification of DIABLO, a mammalian protein that promotes apoptosis by binding to and antagonizing IAP proteins. Cell 102: 43-53

21. Du C, Fang M, Li Y, Li L and Wang X (2000) Smac, a mitochondrial protein that promotes cytochrome $c$-dependent caspase activation by eliminating IAP inhibition. Cell 102: 33-42

22. Lakin ND and Jackson SP (1999) Regulation of p53 in response to DNA damage. Oncogene 18: 7644-7655

23. Hengstschlager M, Braun K, Soucek T, Miloloza A and Hengstschlager-OttnadE (1999) Cyclin-dependent kinases at the G1-S transition of the mammalian cell cycle. Mutat. Res. 436: 1-9

24. Denooij JC and Hariharan IK (1995) Uncoupling cell fate determination from patterned cell division in the Drosophila eye. Science 270: 983-985

25. Hari KL, Santerre A, Sekelsky JJ, McKim KS, Boyd JB and Hawley RS (1995) The mei-41 gene of D. melanogaster is a structural and functional homolog of the human ataxia telangiectasia gene. Cell 82: 815-821
26. Brodsky MH, Sekelsky JJ, Tsang G, Hawley RS and Rubin GM (2000) mus304 encodes a novel DMA damage checkpoint protein required during Drosophila development. Genes Dev. 14: 666-678

27. Banin S, Moyal L, Shieh S, Taya Y, Anderson CW, Chessa L, Smorodinsky NI, Prives C, Reiss Y, Shiloh Y and Ziv Y (1998) Enhanced phosphorylation of p53 by ATM in response to DNA damage. Science 281: 1674-1677

28. Canman CE, Lim DS, Cimprich KA, Taya Y, Tamai K, Sakaguchi K, Appella E, Kastan MB and Siliciano JD (1998) Activation of the ATM kinase by ionizing radiation and phosphorylation of $\mathrm{p} 53$. Science 281: 1677-1679

29. Khanna KK, Keating KE, Kozlov S, Scott S, Gatei M, Hobson K, Taya Y, Gabrielli B, Chan D, Lees-Miller SP and Lavin MF (1998) ATM associates with and phosphorylates p53: mapping the region of interaction. Nat. Genet. 20:398-400

30. Shieh SY, Ikeda M, Taya Y and Prives C (1997) DNA damage-induced phosphorylation of $p 53$ alleviates inhibition by MDM2. Cell 91: 325-334

31. el-Deiry WS, Tokino T, Velculescu VE, Levy DB, Parsons R, Trent JM, Lin D, Mercer WE, Kinzler KW and Vogelstein B (1993) WAF1, a potential mediator of p53 tumor suppression. Cell 75: 817-825

32. Miyashita T, Krajewski S, Krajewska M, Wang HG, Lin HK, Liebermann DA, Hoffman B and Reed JC (1994) Tumor suppressor p53 is a regulator of bcl-2 and bax gene expression in vitro and in vivo. Oncogene 9: 1799-1805

33. Polyak K, Xia Y,Zweier JL, Kinzler KW and Vogelstein B (1997) A model for p53induced apoptosis. Nature 389: 300-305

34. Yu J, Zhang L, Hwang PM, Rago C, Kinzler KW and Vogelstein B (1999) Identification and classification of p53-regulated genes. Proc. Natl. Acad. Sci. USA 96: 14517-14522

35. Zhao R, Gish K, Murphy M, Yin Y, Notterman D, Hoffman WH, Tom E, Mack DH and Levine AJ (2000) Analysis of p53-regulated gene expression patterns using oligonucleotide arrays. Genes Dev. 14: 981-993

36. Tatusova TA and Madden TL (1999) BLAST 2 Sequences, a new tool for comparing protein and nucleotide sequences [published erratum appears in FEMS Microbiol Lett 1999 Aug 1; 177(1): 187-8]. FEMS Microbiol. Lett. 174: $247-250$ 